\title{
APPROXIMATION OF THE AVERAGE WAITING TIME FOR THE HE2/HE2/1 QUEUING SYSTEM USING SIMULATION
}

Kada,Othmane,

PSUTI, Samara, Russia, otman2333@mail.ru
Keywords: Discrete event simulator, queuing system, hypererlangian, average waiting time

In this article we present a simulation based method to approximate the average waiting time for the queuing system (QS) HE2/HE2/I , Which is by Kendall's definition belonging to the class $\mathbf{G} / \mathbf{G} /$, with probabilistic Mixing Distribution of second order hypererlangian distribution inputs for both inter arrival and service time functions Our methods consists of creating a virtual model of Traffic flows, first by using different methods and algorithms of generating random numbers from hypererlangian distribution using a random variate generator and a Discrete event simulator based on a queuing system (QS) of type HE2/HE2/I then use the results to analyse the behavior of the system during different etapes of execution , The results obtained for the average waiting time from our simulation method are very identical to our theoretical results, in the end this work leads us to evaluate accuracy of our theoretical methods. and to collect a big data-set that can be used for other proprieties to find a solution for real problems of modern teletraffic theory.

Information about author:

Kada,Othmane, PhD student, PSUTI, Samara, Russia

Для цитирования:

Када Отхмане. Определение приближенного среднего времени ожидания для системы массового обслуживания HE2/HE2/I с использованием моделирования // Т-Сотm: Телекоммуникации и транспорт. 2020. Том I4. №6. С. 53-60.

For citation:

Kada Othmane. (2020) Approximation of the average waiting time for the HE2/HE2/I queuing system using simulation. T-Comm, vol. I4, no.6, pp. 53-60. (in Russian) 


\section{Introduction}

Traffic flows analysis is a very important part of studying the performance of modern telecommunications network because it gives a better understanding about the resource usage and the average waiting time inside the system which we will study in our work queuing theory techniques involves the mathematical study of traffic flows behavior by modeling it according to the main characteristic of queuing systems which can be described according to Kendall's notation in a basic form by $\mathrm{A} / \mathrm{B} / \mathrm{m}$, where $A$ indicates the distribution of inter-arrivals, $B$ denotes the distribution of service duration, $m$ is the number of servers.

Usually modelisation of traffic flows in modern telecommunications network the $\mathrm{G} / \mathrm{G} / 1$ queuing system is often used where the number of servers is one and a general form of distribution law functions is used, such as erlangian, exponential, hyperexponential, hyperelangian, etc., for which the variation coefficients of random variables are greater than or equal to $1\left(c_{\tau} \geq 1\right)$ or less than $1\left(c_{\tau}<1\right)$.

The coefficient of variation greater than 1 indicates that the probability of the appearance of large values of a random variable is much higher than that of the classical exponential distribution, and the "tail" of the distribution is more powerful. For the coefficient of variation of a smaller one is the opposite, Such system is a typical problem of a discrete event system, and the computer simulation is a quite effective way for analyzing the performances by calculating the average waiting time which From the queuing theory [1-4] in a G/G/1 queue is determined by

$$
\bar{W}=\frac{D_{\lambda}+D_{\mu}+(1-\rho)^{2} / \lambda^{2}}{2(1-\rho) / \lambda}-\frac{I^{2}}{2 \bar{I}}
$$

where $\rho$ is the system load factor $(0<\rho=\lambda / \mu<1), \lambda$ is the intensity of the input stream, $\mu$ is the service intensity, $D_{\lambda}, D_{\mu}$ respectively the variance of the arrival intervals and the service time, $\bar{I}, \overline{I^{2}}$ respectively the average value and the second initial moment of the idle period in this paper we will present a different way to approximate the average waiting time in the queuing system by creating a virtual traffic flow using a Discrete Event Simulator.

\section{Stateme of the problem}

Our task is to find the average waiting time of the HE2 / HE2 / 1, for this the classical method of spectral decomposition of the solution of the Lindley integral equation is widely used but for our case, the problem is that when studying such systems as $\mathrm{HE} 2 / \mathrm{HE} 2 / 1$ (QS) is difficult to find a solution for the average waiting time due to high computational complexity, that is why we will use a simulation based solution to approximate the average waiting time of this queuing system.

\section{III. solution of the problem}

We take the $\mathrm{HE}_{2} / \mathrm{HE}_{2} / 1$ (QS) with probabilistic density functions (pdf) in the inputs.

inter-arrivals function:

$$
a(t)=4 p \lambda_{1}^{2} t e^{-2 \lambda_{1} t}+4(1-p) \lambda_{2}^{2} t e^{-2 \lambda_{2} t},
$$

service time function:

$$
b(t)=4 p \mu_{1}^{2} t e^{-2 \mu_{1} t}+4(1-p) \mu_{2}^{2} t e^{-2 \mu_{2} t} .
$$

\section{Inverse-transform Technique}

The inverse-transform technique can be used in principle for any distribution having density functions (pdf) $\mathrm{f}(\mathrm{x})$. And Most useful when the Cumulative distribution function CDF F(x) has an inverse $\mathrm{F}-1(\mathrm{x})$ which is easy to compute. It consists of 4 steps example on Exponential distribution.

Take the exponential distribution function with the PDF $f(X)=\lambda e^{-x \lambda}$.

1. find the $\mathrm{CDF} F(X)$ by integrate the PDF $f(X)$,

$$
\begin{aligned}
& F(X)=\int_{-\inf }^{X} f(X): \\
& F(X)=\int_{-\inf }^{X} f(X)=1-e^{-x \lambda}
\end{aligned}
$$

2. Set $\mathrm{F}(\mathrm{X})=\mathrm{R}$ on the range of $\mathrm{X}: 1-e^{-x \lambda}=R$.

3. Solve the equation $F(X)=R$ for $X$ in terms of $R$ :

$$
X=\frac{-1}{\lambda} \ln (1-R) \text {. }
$$

4. nerate uniform random numbers $\mathrm{R}_{1}, \mathrm{R}_{\mathrm{i}}$ and compute the random variate by $X_{i}=F^{-1}\left(R_{i}\right)$ where $R_{i}$ is a uniformly distributed random number on $(0,1)$ exponential variate can be generated by

$$
X_{i}=\frac{-\ln \left(1-R_{i}\right)}{\lambda} \text {. }
$$

Example of anexponential variate generator algorithm.

Function $\operatorname{Rexp}(\lambda)$ :

$$
\mathrm{R}=\operatorname{random}()
$$$$
\text { return }(-\ln (1-\mathrm{R}) / \lambda)
$$

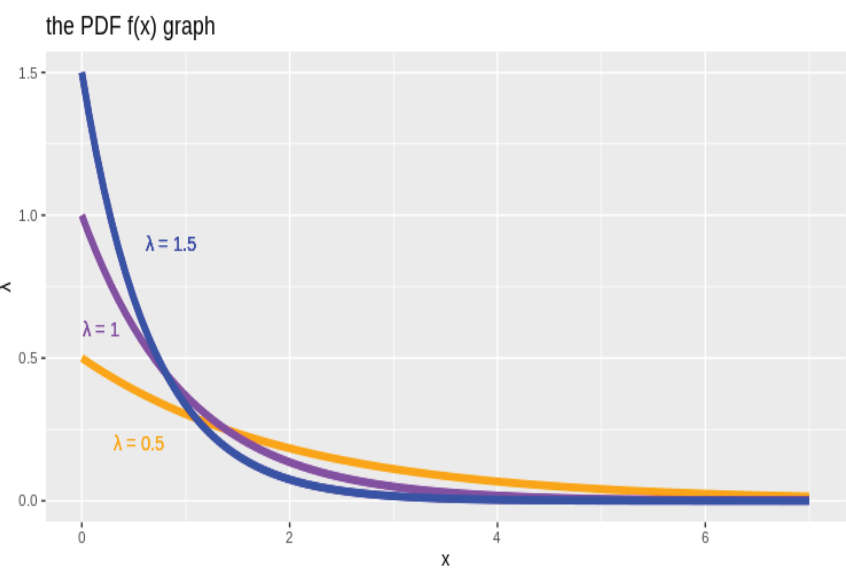

Fig. 1. Exponential PDF graphs

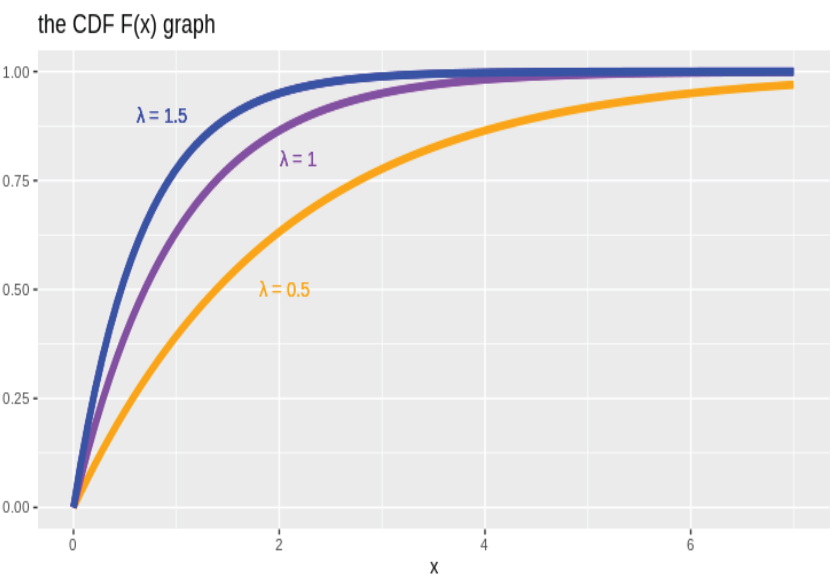

Fig. 2. Exponential CDF graphs 


\section{Convolution Generation Technique}

The distribution of the sum of two or more random variables is called the convolution. Let $\mathrm{Y}_{\mathrm{i}}<\mathrm{G}(\mathrm{y})$ be IID random variables $Y=\sum_{i=1}^{n} X_{i}$. Then the distribution of $\mathrm{Y}$ is said to be the convolution of $X_{\mathrm{i}}$.

Example of erlang distribution:

take $Y_{1}$ and $Y_{2}$ two exponential distribution function with the rate $\lambda=2 \lambda$ where:

$$
\begin{aligned}
& \left(X_{1}\right)=2 \lambda e^{-x(2 \lambda)},\left(X_{2}\right)=2 \lambda e^{-x(2 \lambda)} \\
& Y=\left(X_{1}\right)+\left(X_{2}\right)=2 \lambda e^{-x(2 \lambda)}+2 \lambda e^{-x(2 \lambda)} \\
& \text { lets find the CDF of } X \\
& C D F(Y)=P\left(X_{1}+X_{2} x\right) \\
& C D F(Y)=\int_{x_{1}} P\left(X_{1}+X_{2} \quad x \vee X_{1}\right) \cdot P\left(X_{1}\right) d x_{1} \\
& C D F(Y)=\int_{0}^{x} P\left(X_{2} x-X_{1}\right) \cdot 2 \lambda e^{-x_{1} \cdot(2 \lambda)} d x_{1} \\
& C D F(Y)=\int_{0}^{x} P\left(X_{2} x-X_{1}\right) \cdot 2 \lambda e^{-x_{1} \cdot(2 \lambda)} d x_{1} \\
& C D F(Y)=\int_{0}^{x}\left(1-e^{-2 \lambda\left(x-X_{1}\right)}\right) 2 \lambda e^{-2 \lambda X_{1}} d x_{1} \\
& C D F(Y)=1-e^{-2 \lambda x}-2 \lambda x e^{-2 \lambda x}
\end{aligned}
$$

Then finding the PDF from the CDF:

$$
\begin{aligned}
& P D F(Y)=\frac{d x}{x} P D F(Y)=\frac{d x}{x}\left(1-e^{-2 \lambda x}-2 \lambda x e^{-2 \lambda x}\right) \\
& P D F(Y)=4 \lambda^{2} x e^{-2 \lambda x} .
\end{aligned}
$$

Got the distribution function with the PDF $f(X)=$ $4 \lambda^{2} x e^{-2 \lambda x}$ and $\operatorname{CDF} F(x)=1-e^{-2 \lambda x}-2 \lambda x e^{-2 \lambda x}$ which is an Erlang distribution with shape 2 and rate $=2 \lambda$, that means that if $\mathrm{Y}$ is Erlang distributed with parameters ( then $Y$ can be expressed as a sum of independent exponential $\mathrm{X}_{\mathrm{i}}$ with the shape of $\mathrm{K}$, so Erlang variate can be generated with $Y_{i}=\sum_{1=1}^{K} \frac{-\ln \left(1-R_{i}\right)}{\lambda K}$ in the case of $k=2$ and rate $=2 \lambda$, $Y_{i}=\sum_{1=1}^{2} \frac{-\ln \left(1-R_{i}\right)}{4 \lambda}$.

Example of an Erlang variate generator algorithm.

Function Rerlang $(\lambda, k)$ :

$\mathrm{s}=0$

for (i from 1 to $\mathrm{k}$ ) do:

return( $\mathrm{S}$ )

$$
\mathrm{s}=\mathrm{s}+\operatorname{Rexp}\left(\lambda^{*} \mathrm{k}\right)
$$

the PDF $f(x)$ graph

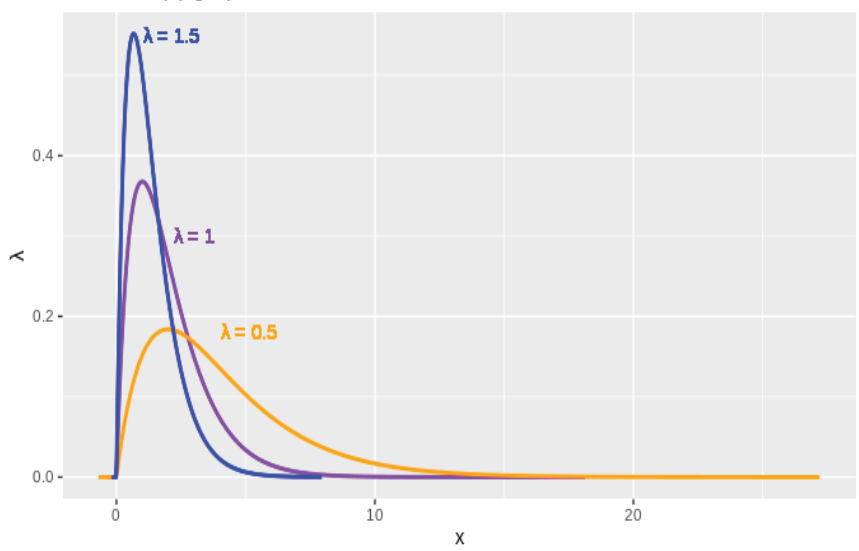

Fig. 3. Erlang PDF graphs

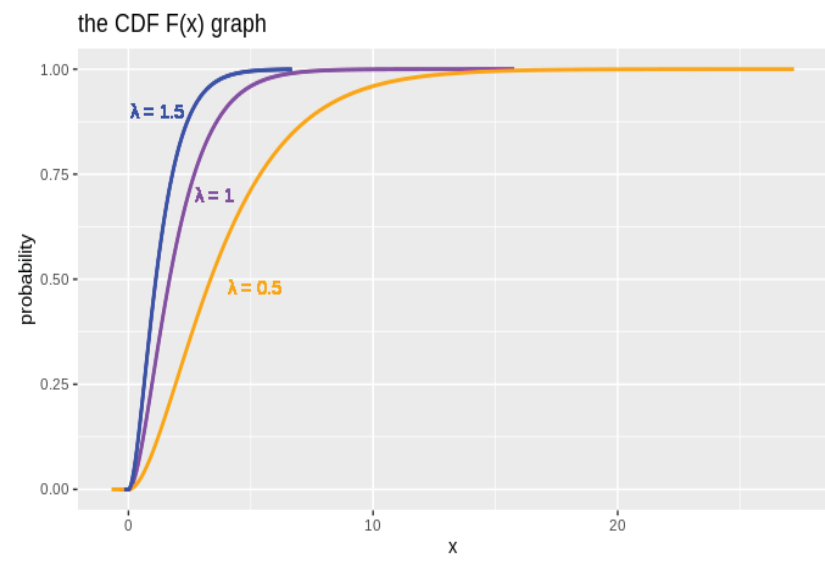

Fig. 4. Erlang CDF graphs

\section{Composition Technique}

This method applies when the distribution function $\mathrm{F}$ can be expressed as a mixture of other distribution functions $\mathrm{F}_{1}, \mathrm{~F}_{2}, \mathrm{~F}_{\mathrm{i}}$

$F_{i}=\sum_{1=1}^{K} p_{i} F_{i}$ where $p_{i} \geq 0, \sum_{1=1}^{\infty} p_{i}=1$.

This method is useful if it is easier to sample from the $F_{i}$ than from $\mathrm{F}$.

First generate an index $I$ such that $P(I=i)=p_{i}, i=1,2$. Then Generate a random variable $\mathrm{X}$ with distribution function $\mathrm{F}_{\mathrm{I}}$.

Example of hyper erlang distribution.

Take $\mathrm{HE}_{2}$ the probabilistic mixture of two erlang distribution of second order with rate $=2 \lambda$

$E_{2}(x)=4 \lambda^{2} x e^{-2 \lambda x} H E_{2}=\sum_{1=1}^{2} p_{i} E_{2}$

where $p_{i} \geq 0, p_{1}=p, p_{2}=(1-p)$

$H E_{2}(x)=4 p \lambda_{1}^{2} x e^{-2 \lambda_{1} x}+4(1-p) \lambda_{2}^{2} x e^{-2 \lambda_{2} x}$

$C D F\left(H E_{2}\right)=p\left(1-e^{-2 \lambda_{1} x}-2 \lambda_{1} x e^{-2 \lambda_{1} x}\right)+$

$+(1-p)\left(1-e^{-2 \lambda_{2} x}-2 \lambda_{2} x e^{-2 \lambda_{2} x}\right)$.

Hyper Erlang with shapes $(2,2)$ and rates $\left(2 \lambda_{1}, 2 \lambda_{2}\right)$ and probabilistic mixtures $(\mathrm{p}, 1-\mathrm{p})$ variate with can be generated with $H E_{i}=p \sum_{1=1}^{2} \frac{-\ln \left(1-R_{i}\right)}{4 \lambda_{1}}+(1-p) \sum_{1=1}^{2} \frac{-\ln \left(1-R_{i}\right)}{4 \lambda_{2}}$.

Example of an hyper Erlang variate generator algorithm. Function RhyperErlang (V $\lambda, \mathrm{Vp}, \mathrm{Vk})$ :

$\mathrm{s}=0$

for (i from 1 to size $(V p))$ do:

for ( $\mathrm{j}$ from 1 to size $(\mathrm{V} \lambda))$ do: $\mathrm{s}=\mathrm{s}+$ Rerlang $\left(\mathrm{V} \lambda_{\mathrm{j}}, \mathrm{Vk}_{\mathrm{i}}\right)$

return( $\mathrm{S}$ ) $\mathrm{s}=\mathrm{s}^{*} \mathrm{Vp} \mathrm{p}_{\mathrm{i}}$

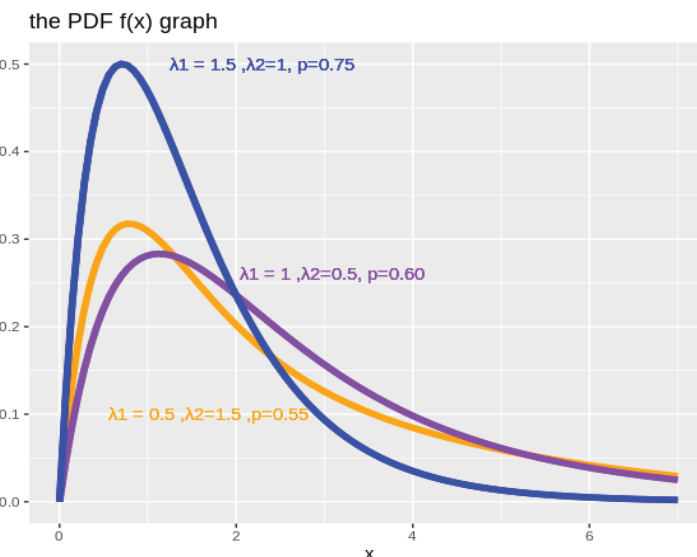

Fig. 5. Hyper Erlang PDF graphs 


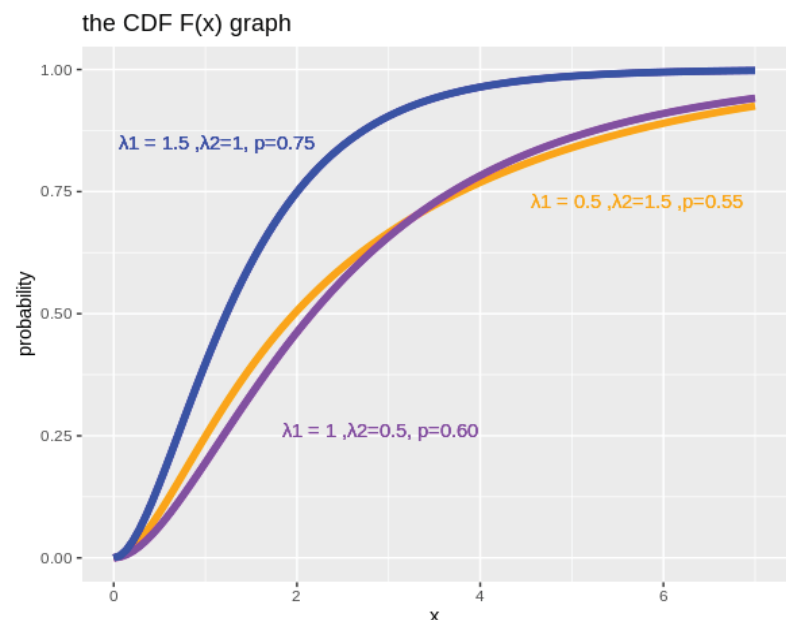

Fig. 6. Hyper Erlang CDF graphs

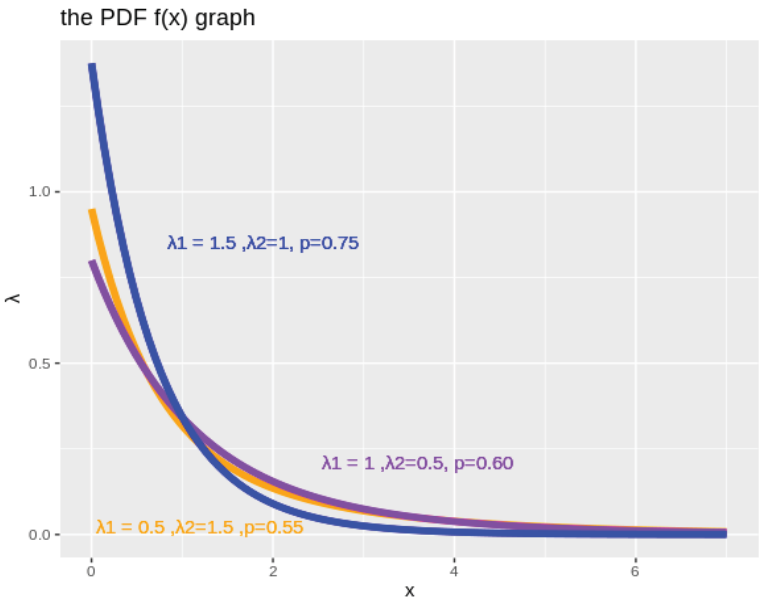

Fig. 7. Hyper Exponential PDF graphs

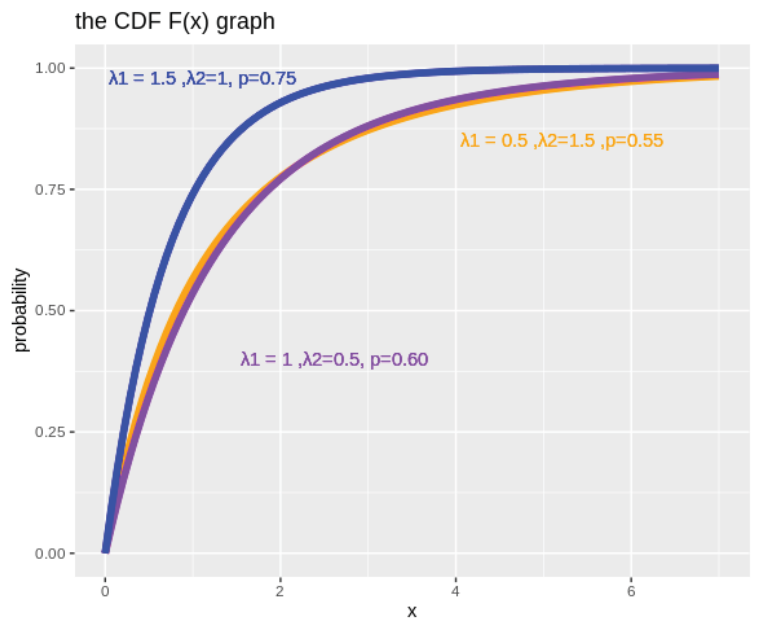

Fig. 8. Hyper Exponential CDF graphs

Example of hyper erlang distribution.

Take $\mathrm{H}_{2}$ the probabilistic mixture of two exponential distribution of second order with rate $=\lambda$

$$
\begin{aligned}
& H(x)=\lambda x e^{-\lambda x} \\
& H_{2}=\sum_{1=1}^{2} p_{i} H \text { where } p_{i} \geq 0, p_{1}=p, p_{2}=(1-p) \\
& H_{2}(x)=p \lambda_{1} x e^{-\lambda_{1} x}+(1-p) \lambda_{2} x e^{-\lambda_{2} x} \\
& C D F\left(H_{2}\right)=p\left(1-e^{-\lambda_{1} x}\right)+(1-p)\left(1-e^{-\lambda_{2} x}\right)
\end{aligned}
$$

Hyper exponential with rates $\left(\lambda_{1}, \lambda_{2}\right)$ and probabilistic mixtures $(\mathrm{p}, 1-\mathrm{p})$ variate can be generated with

$$
H_{i}=p \frac{-\ln \left(1-R_{i}\right)}{\lambda_{1}}+(1-p) \frac{-\ln \left(1-R_{i}\right)}{\lambda_{2}} .
$$

Example of an hyper Erlang variate generator algorithm.

Function RhyperExp (Vג,Vp):

$$
\begin{aligned}
& \mathrm{s}=0 \\
& \text { for (i from } 1 \text { to size }(\mathrm{Vp})) \text { do: } \\
& \text { for }(\mathrm{j} \text { from } 1 \text { to size }(\mathrm{V} \lambda)) \text { do: } \\
& \qquad \mathrm{s}=\mathrm{s}+\operatorname{Rexp}\left(\mathrm{V} \lambda_{\mathrm{j}}\right) \\
& \mathrm{s}=\mathrm{s}^{*} \mathrm{~V} \mathrm{p}_{\mathrm{i}}
\end{aligned}
$$

\section{Discrete event simulator}

Discrete Event Simulation (DES) is the simulation that modelate a complex system behavior an ordered sequence of discrete events collection, when each event have a known effect on the rest of the system state at a specific point in time.

Each process inside the system can defined based on their impact on the system, their resource requirements, and their trigger way, may be scheduled or occurred randomly, or occurred responsively to other event in the system, in the end they all will be combined within the simulation to modelate the system from the scratch application on queuing system:

In the queuing system model two types of events are used, arrival and departure. The arrival is when a customer reaches a service station, and the departure corresponds to the event when the customer leaves the system. And logically the arrival event for a customer is executed before its departure event example of $\mathrm{HE}_{2} / \mathrm{HE}_{2} / 1$ queue simulation algorithm:

function queue (arrivals,servicing, $\mathrm{n}, \mathrm{k}$ )

$$
\mathrm{q}=[]
$$

for $\mathrm{i}$ (from1 to $\mathrm{n})$ do:

$$
\mathrm{a}_{\mathrm{i}}=\text { RhyperErlang(arrivals) }
$$$$
\mathrm{b}_{\mathrm{i}}=\text { RhyperErlang(servicing) }
$$

$\mathrm{a}=\operatorname{cumsum}\left(\mathrm{a}_{\mathrm{i}}\right)$

$\mathrm{k}=1$

$\mathrm{q}=\operatorname{append}(\mathrm{q}, \operatorname{steps}(\mathrm{a}, \mathrm{b}, \mathrm{k}, \mathrm{n}))$

function steps $(\mathrm{a}, \mathrm{b}, \mathrm{k}, \mathrm{n})$

Sort $(\mathrm{a}, \mathrm{b})$ in terms of a(ascending)

Create vector $\mathrm{p}_{\mathrm{n}}$

Create vector $\mathrm{s}_{\mathrm{R}}$

Create vector $d_{n}$ $\mathrm{s}_{\mathrm{k}}=0$

$$
\begin{aligned}
& \text { for } \mathrm{i} \text { (from1 to } \mathrm{n} \text { ) do } \\
& \qquad \begin{array}{l}
\mathrm{p}=\arg \min (\mathrm{s}) \\
\mathrm{sp}_{\mathrm{i}}=\max \left(\mathrm{a}_{\mathrm{i}}, \mathrm{sp}_{\mathrm{i}}\right)+\mathrm{b}_{\mathrm{i}} \\
\mathrm{d}_{\mathrm{i}}=\mathrm{sp}_{\mathrm{i}}
\end{array}
\end{aligned}
$$

end for

Put (a,d,p) back to original (input) ordering of a return $(\mathrm{d}, \mathrm{p})$

\section{Results}

We made our simulation based $\mathrm{R}$ language using RStudio and a list of useful 1 libraries .Distr :an Object Oriented Implementation of Distributions, Simmer: a process-oriented and trajectory-based Discrete-Event Simulation, Parallel: Support for Parallel computation.

To start simulation we have as an inputs inter-arrivals function (1) with the variables $\left(\lambda_{1}, \lambda_{2}, p\right)$ and the servicing function (2) 
with the variables $\left(\mu_{1}, \mu_{2}, q\right)$, choosing the best combination value of the variables to get the best simulation experience is very important that why we used the combination from our theoretical solution to the approximation to the average waiting time to the queuing system $\mathrm{HE}_{2} / \mathrm{HE}_{2} / 1$ [2], where we have:

$$
\begin{aligned}
& \lambda_{1}=2 p / \bar{\tau}_{\lambda}, \quad p=\frac{1}{2} \pm \sqrt{\frac{2\left(1+c_{\lambda}^{2}\right)-3}{8\left(1+c_{\lambda}^{2}\right)}} \\
& \mu_{1}=2 q / \bar{\tau}_{\mu}, \quad \mu_{2}=2(1-q) / \bar{\tau}_{\mu}, \quad q=\frac{1}{2}\left(1 \pm \sqrt{\frac{c_{\mu}^{2}-1}{c_{\mu}^{2}+1}}\right) \\
& \rho=\bar{\tau}_{\mu} / \bar{\tau}_{\lambda}, \quad \bar{\tau}_{\mu}=1
\end{aligned}
$$

where $\rho$ refers to the server utilization, take

Table 1

Inputs combination for $\mathrm{a}(\mathrm{t})$ and $\mathrm{b}(\mathrm{t})$

\begin{tabular}{|c|c|c|c|}
\hline$\rho$ & $\left(c_{\lambda}, c_{\mu}\right)$ & $\left(\lambda_{1}, \lambda_{2}, \mathrm{p}\right)$ & $\left(\mu_{1}, \mu_{2}, \mathrm{q}\right)$ \\
\hline \multirow{3}{*}{0,1} & & $(0.1052210$, & $(1.052210$, \\
& $(0,71 ; 0,71)$ & 0.094779001, & 0.94779001, \\
& & $0.5261050)$ & $0.5261050)$ \\
\hline \multirow{3}{*}{0,5} & \multirow{2}{*}{$0,71 ; 0,71)$} & $(0.5261050$, & $(1.052210$, \\
& & 0.473895007, & 0.94779001, \\
& & $0.5261050)$ & $0.5261050)$ \\
\hline \multirow{3}{*}{0.9} & \multirow{2}{*}{$(0,71 ; 0,71)$} & $(0.9469890$, & $(1.052210$, \\
& & 0.853011013, & 0.94779001, \\
& & $0.5261050)$ & $0.5261050)$ \\
\hline
\end{tabular}

Replacing $\left(\lambda_{1}, \lambda_{2}, \mathrm{p}\right)$ in $\mathrm{a}(\mathrm{t})(1)$ and $\left(\mu_{1,}, \mu_{2}, \mathrm{q}\right)$ in $\mathrm{b}(\mathrm{t})(2)$ we get:

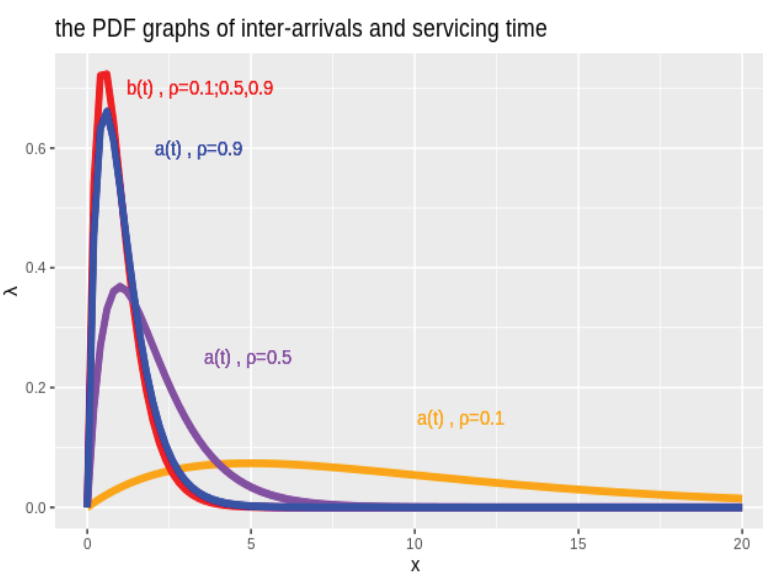

Fig. 9. $P D F$ graphs for $a(t)$ and $b(t)$

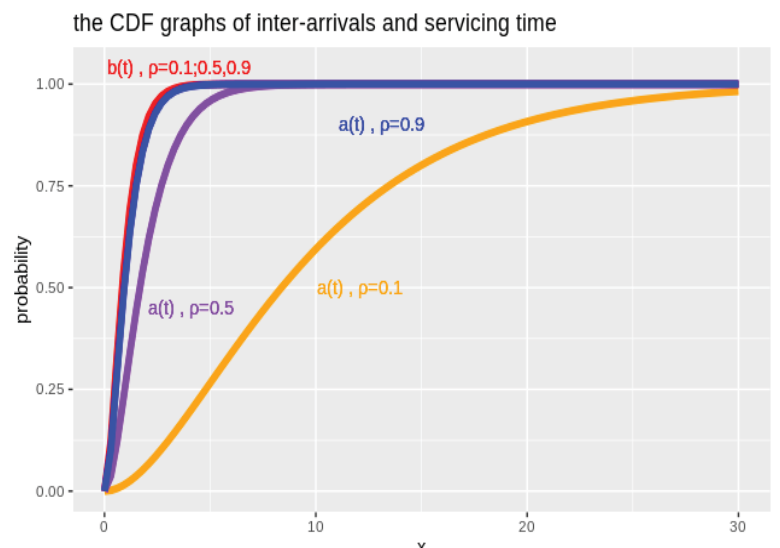

Fig. 10. CDF graphs for $a(t)$ and $b(t)$

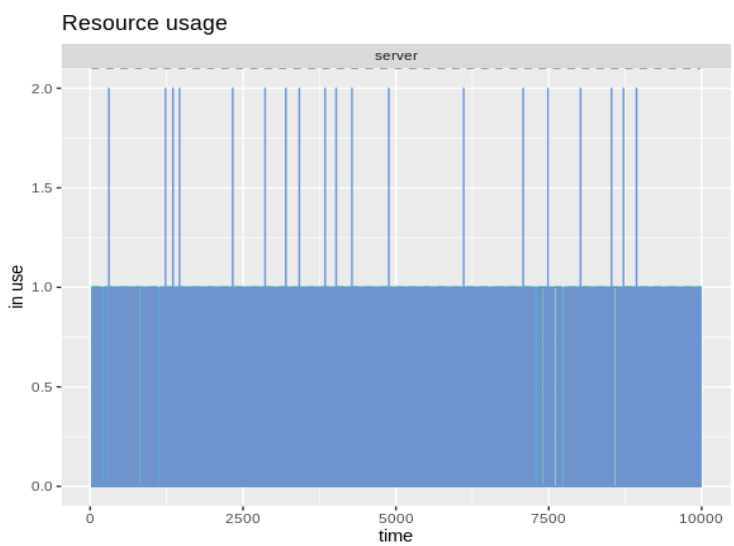

Fig. 11. Resource Usage by step, $\rho=0.1$

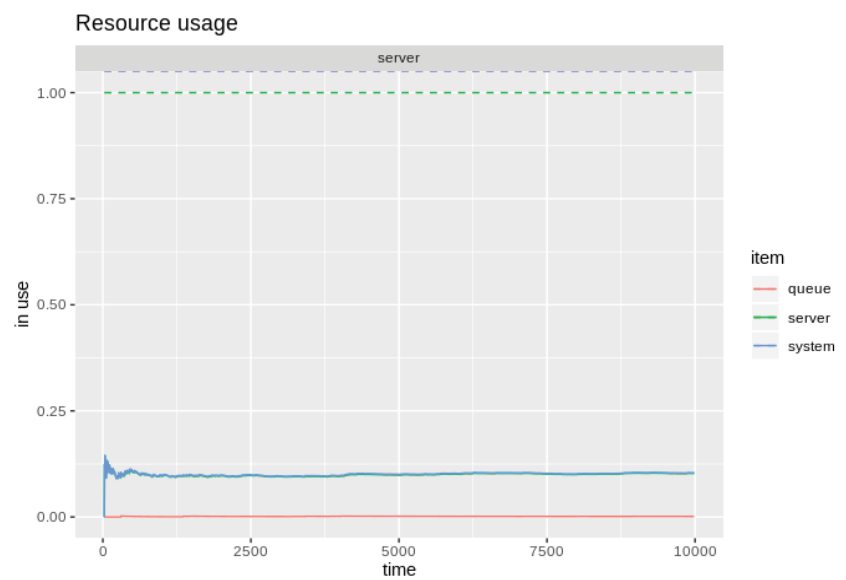

Fig. 12. Resource Usage, $\rho=0.1$

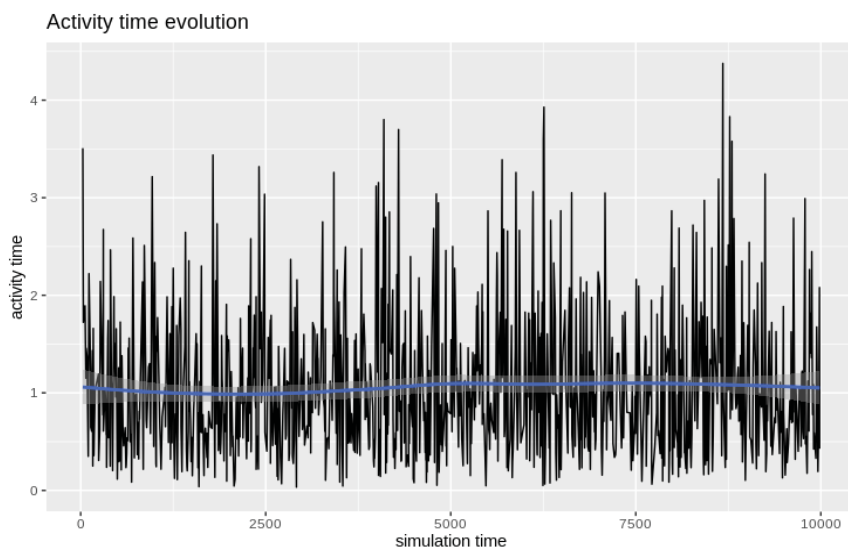

Fig. 13. Servicing time, $\rho=0.1$

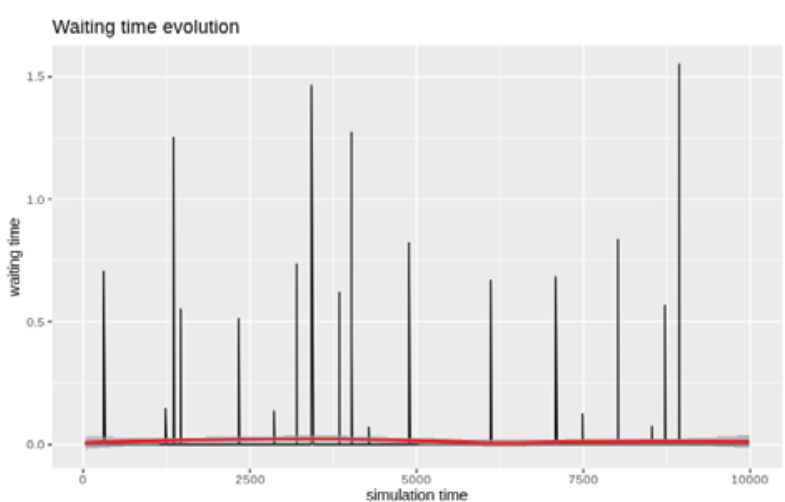

Fig. 14. Waiting time, $\rho=0.1$ 


\begin{tabular}{|c|c|c|c|c|c|c|c|}
\hline & name $*$ & start time $=$ & end_time & activity_time & finished & replication & waiting_time $\stackrel{*}{*}$ \\
\hline 1 & arrivalo & 24.91055 & 28.41832 & 3.50776938 & TRUE & 1 & $8.881784 \mathrm{e}-16$ \\
\hline 2 & arrival1 & 34.44206 & 36.16511 & 1.72305177 & TRUE & 1 & $0.000000 \mathrm{e}+00$ \\
\hline 3 & arrival2 & 56.87647 & 58.76803 & 1.89155824 & TRUE & 1 & $1.776357 \mathrm{e}-15$ \\
\hline 4 & arrival3 & 69.08397 & 70.23390 & 1.14992583 & TRUE & 1 & 4.440892e-16 \\
\hline 5 & arrival4 & 71.83395 & 73.29041 & 1.45645337 & TRUE & 1 & $0.000000 e+00$ \\
\hline 6 & arrival5 & 84.85442 & 86.21450 & 1.36007235 & TRUE & 1 & $5.107026 \mathrm{e}-15$ \\
\hline 7 & arrival6 & 101.94744 & 102.29720 & 0.34976099 & TRUE & 1 & $3.497203 \mathrm{e}-15$ \\
\hline 8 & arrival7 & 109.66216 & 111.88341 & 2.22125130 & TRUE & 1 & $1.332268 \mathrm{e}-15$ \\
\hline 9 & arrivals & 132.87503 & 134.05040 & 1.17537088 & TRUE & 1 & $9.992007 \mathrm{e}-15$ \\
\hline 10 & arrival9 & 135.14059 & 135.78928 & 0.64869888 & TRUE & 1 & $9.325873 \mathrm{e}-15$ \\
\hline 11 & arrival10 & 148.38461 & 148.99106 & 0.60644145 & TRUE & 1 & $5.218048 \mathrm{e}-15$ \\
\hline
\end{tabular}

Fig. 15. Head of queuing results table, $\rho=0.1$

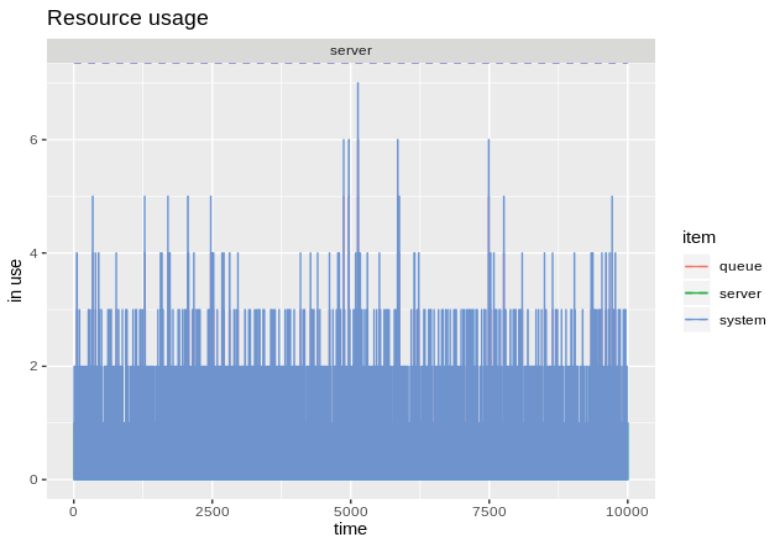

Fig. 16. Resource Usage by step, $\rho=0.5$

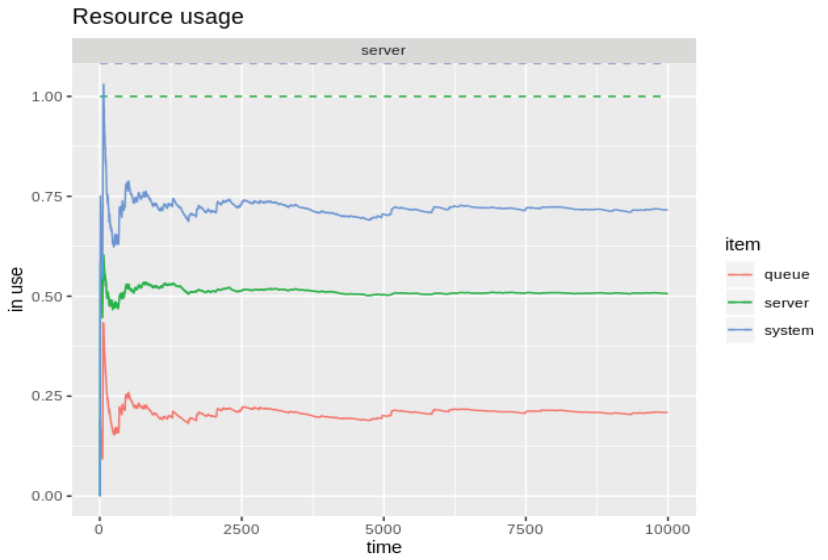

Fig. 17. Resource Usage, $\rho=0.5$

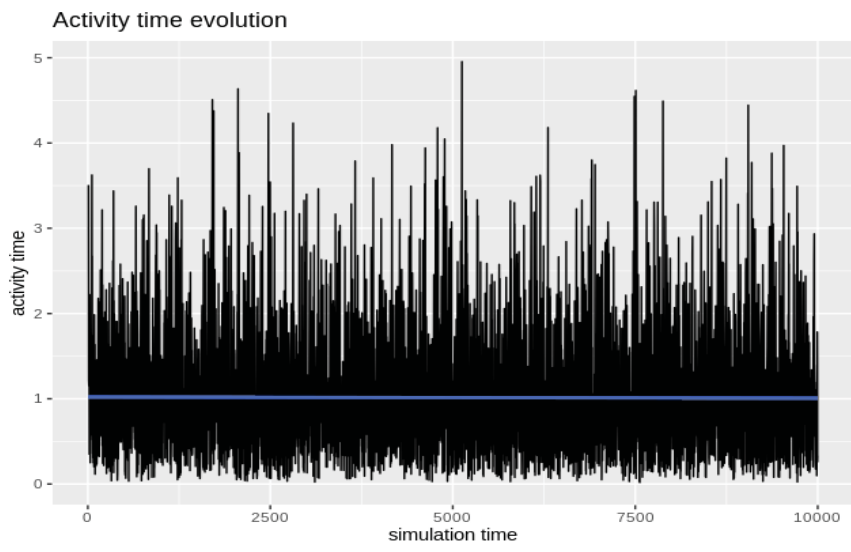

Fig. 18. Servicing time, $\rho=0.5$

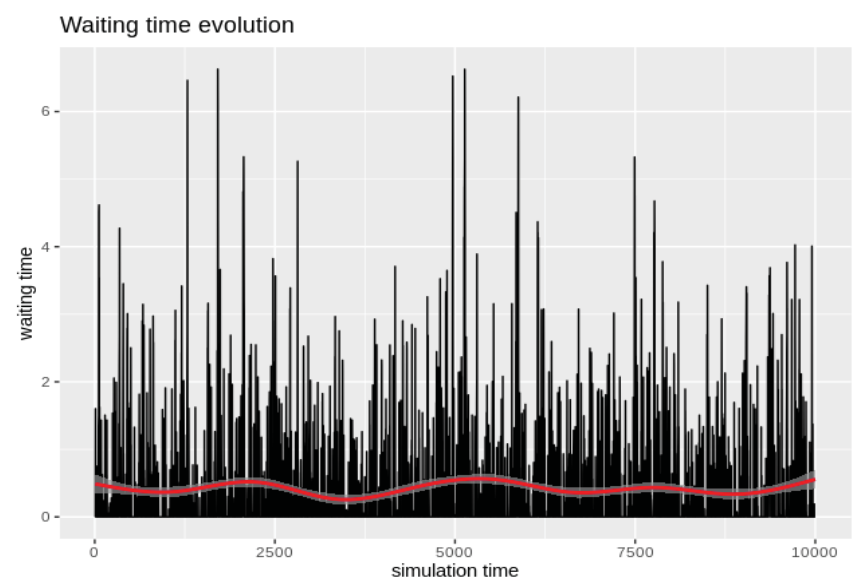

Fig. 19. Waiting time, $\rho=0.5$

\begin{tabular}{|c|c|c|c|c|c|c|c|}
\hline . & name & $\neq$ start time & end_time & activity_time & finished & ث replication & Waiting_time \\
\hline 1 & arrivalo & 4.982109 & 8.489879 & 3.50776938 & TRUE & 1 & $8.881784 \mathrm{e}-16$ \\
\hline 2 & arrival1 & 6.888412 & 10.733320 & 2.24344149 & TRUE & 1 & $1.601467 \mathrm{e}+00$ \\
\hline 3 & arrival2 & 10.334515 & 11.954070 & 1.22074993 & TRUE & 1 & $3.988046 \mathrm{e}-01$ \\
\hline 4 & arrival3 & 14.117632 & 15.267558 & 1.14992583 & TRUE & 1 & $4.440892 \mathrm{e}-16$ \\
\hline 5 & arrival4 & 14.667628 & 16.569605 & 1.30204708 & TRUE & 1 & $5.999297 \mathrm{e}-01$ \\
\hline 6 & arrival5 & 17.580535 & 18.940607 & 1.36007235 & TRUE & 1 & $1.554312 \mathrm{e}-15$ \\
\hline 7 & arrival6 & 20.999138 & 21.348899 & 0.34976099 & TRUE & 1 & $0.000000 \mathrm{e}+00$ \\
\hline 8 & arrival7 & 22.542082 & 24.763333 & 2.22125130 & TRUE & 1 & $1.332268 \mathrm{e}-15$ \\
\hline 9 & arrival8 & 27.184657 & 28.360027 & 1.17537088 & TRUE & 1 & $0.000000 e+00$ \\
\hline 10 & arrival9 & 27.637767 & 29.684430 & 1.32440282 & TRUE & 1 & $7.222605 \mathrm{e}-01$ \\
\hline
\end{tabular}

Fig. 20. Head of queuing results table, $\rho=0.5$

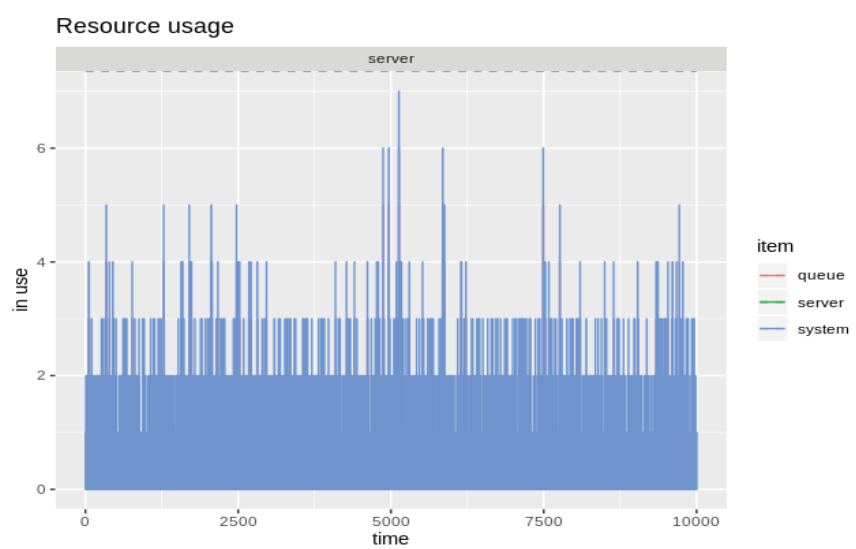

Fig. 21. Resource Usage by step, $\rho=0.9$

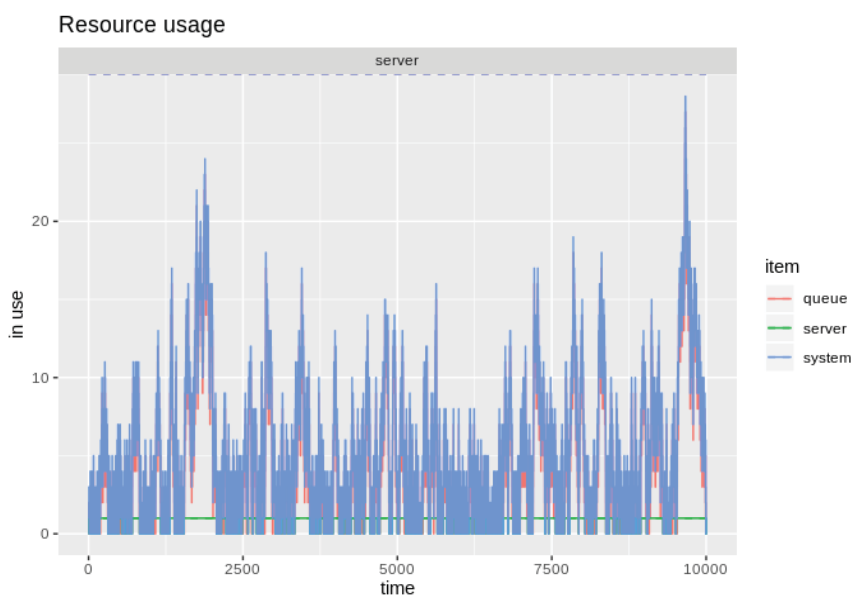

Fig. 22. Resource Usage, $\rho=0.9$ 


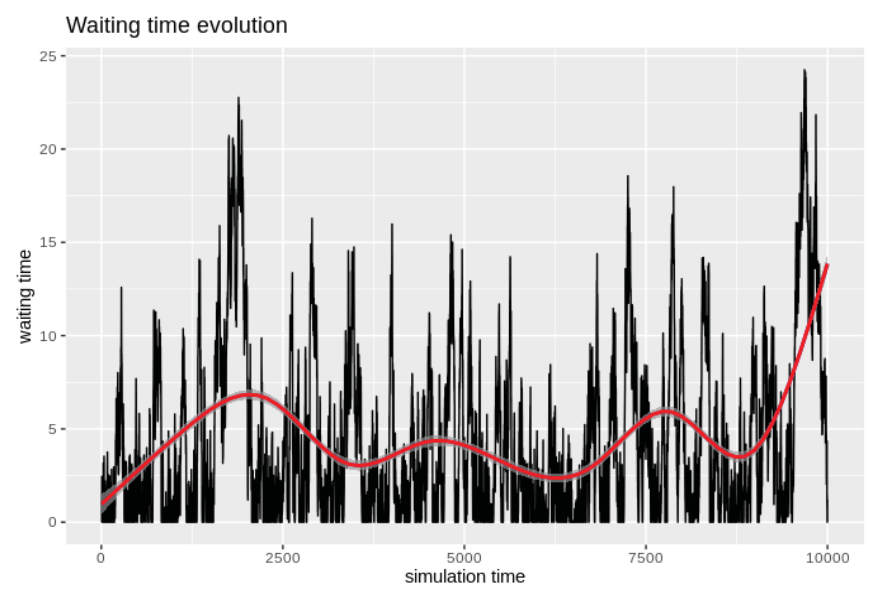

Fig. 23. Servicing time, $\rho=0.9$

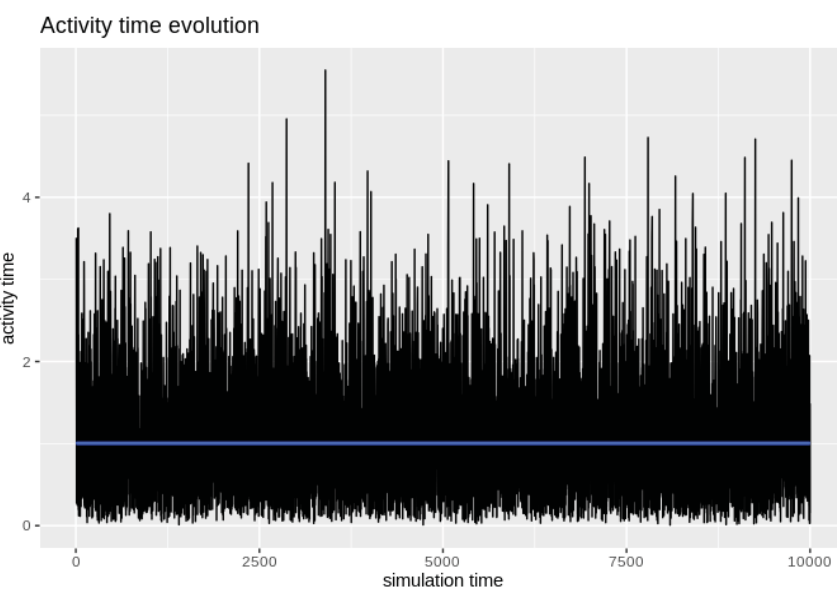

Fig. 24. Waiting time, $\rho=0.9$

\begin{tabular}{|c|c|c|c|c|c|c|}
\hline name & start time $\hat{\imath}$ & er & activity_time & & :ation & \\
\hline arrivalo & 2.767838 & 6.275608 & 3.50776938 & TRUE & 1 & 4.440892e-16 \\
\hline 2 arrivall & 3.826896 & 8.167166 & 1.89155824 & TRUE & 1 & $2.448712 \mathrm{e}+00$ \\
\hline 3 arrival2 & 5.741397 & 9.387916 & 1.22074993 & TRUE & 1 & $2.425769 \mathrm{e}+00$ \\
\hline 4 arrival3 & 8.234110 & 9.662914 & 0.27499805 & TRUE & 1 & $1.153806 \mathrm{e}+00$ \\
\hline 5 arrival4 & 9.511806 & 10.964961 & 1.30204708 & TRUE & 1 & $1.511084 \mathrm{e}-01$ \\
\hline 6 arrival5 & 11.130087 & 12.490159 & 1.36007235 & TRUE & 1 & $0.000000 e+00$ \\
\hline 7 arrival6 & 13.029311 & 13.379072 & 0.34976099 & TRUE & 1 & $0.000000 e+00$ \\
\hline 8 arrival7 & 13.886502 & 16.107753 & 2.22125130 & TRUE & 1 & $0.000000 \mathrm{e}+00$ \\
\hline 9 arrival8 & 16.465710 & 17.641081 & 1.17537088 & TRUE & 1 & $0.000000 e+00$ \\
\hline arrival9 & 16.717438 & 18.247523 & 0.60644145 & TRUE & 1 & $9.236429 \mathrm{e}-01$ \\
\hline arrival10 & 17.438215 & 19.199754 & 0.95223093 & TRUE & 1 & $8.093078 \mathrm{e}-01$ \\
\hline 2 arrival11 & 18.909774 & 19.738379 & 0.53862530 & TRUE & 1 & 2.899801e-01 \\
\hline
\end{tabular}

Fig. 25. Head of queuing results table, $\rho=0.9$

Table 2 show the comparison between simulation and theoretical results of the average waiting time for the $\mathrm{H}_{2} / \mathrm{H}_{2} / 1$ and $\mathrm{HE}_{2} / \mathrm{HE}_{2} / 1$ systems for the cases of low, medium and high loads $\rho=0,1 ; 0,5 ; 0,9$

Here we take into account that the variation range of variation coefficients of the arrivals intervals of input flow $c_{\lambda}$ and service time $c_{\mu}$ for the distribution of $\mathrm{HE}_{2}$ is wider than that of the distribution of $\mathrm{H}_{2}$. Taking into account that the $\mathrm{H}_{2} / \mathrm{H}_{2} / 1$ system is not applicable in cases $c_{\lambda}<1$ and $c_{\mu}<1$ to evaluate our simulation results we made a comparison with our theoretical results using mean absolute percentage error (MAPE) for calculate the loss and the Coefficient of Determination R-Squared for the score.

Table 2

\begin{tabular}{|c|c|c|c|c|c|}
\hline \multicolumn{2}{|c|}{\begin{tabular}{|l|}
$\begin{array}{l}\text { Inputs } \\
\text { ters }\end{array}$ \\
\end{tabular}} & \multicolumn{2}{|c|}{$\begin{array}{l}\text { Average Waiting Time for } \\
\mathrm{QS} \mathrm{H}_{2} / \mathrm{H}_{2} / 1\end{array}$} & \multicolumn{2}{|c|}{$\begin{array}{l}\text { Average Waiting Time } \mathrm{f} \\
\mathrm{QS} \mathrm{HE}_{2} / \mathrm{HE}_{2} / 1\end{array}$} \\
\hline$\rho$ & $\left(c_{i}, C_{i}\right)$ & $\begin{array}{l}\text { theoretical } \\
\text { method }\end{array}$ & $\begin{array}{l}\text { simulation } \\
\text { method }\end{array}$ & $\begin{array}{l}\text { theoretical } \\
\text { method }\end{array}$ & $\begin{array}{l}\text { simulation } \\
\text { method }\end{array}$ \\
\hline \multirow{4}{*}{0,1} & $\begin{array}{l}(0,71 \\
0,71) \\
\end{array}$ & - & & 0,02 & 0.02 \\
\hline & $(2,2)$ & 0,445 & 0.456 & 0,34 & 0.33 \\
\hline & $(4,4)$ & 1,779 & 1.912 & 1,68 & 1.81 \\
\hline & $(8,8)$ & 7,112 & 7.749 & 7,16 & 8.13 \\
\hline \multirow{4}{*}{0,5} & $\begin{array}{l}(0,71 \\
0,71)\end{array}$ & - & & 0,401 & 0.40 \\
\hline & $(2,2)$ & 4,044 & 4.112 & 3,98 & 4.01 \\
\hline & $(4,4)$ & 16,129 & 16.622 & 16,53 & 16.63 \\
\hline & $(8,8)$ & 64,178 & 74.01 & 66,73 & 65.30 \\
\hline \multirow{4}{*}{0,9} & $\begin{array}{l}(0,71 \\
0,71) \\
\end{array}$ & - & & 4,299 & 4.35 \\
\hline & $(2,2)$ & 36,20 & 36.465 & 36,21 & 35.90 \\
\hline & $(4,4)$ & 144,833 & 150.330 & 145,31 & 135.10 \\
\hline & $(8,8)$ & 577,861 & 655.579 & 580,56 & 570.20 \\
\hline
\end{tabular}

Table 3

Evaluation of the result from simulation results

\begin{tabular}{|c|c|c|c|c|}
\hline $\begin{array}{c}\text { Inputs } \\
\text { parameters }\end{array}$ & \multicolumn{2}{|c|}{$\begin{array}{c}\text { Average Waiting Time } \\
\text { for QS } \mathrm{H}_{2} / \mathrm{H}_{2} / 1\end{array}$} & $\begin{array}{c}\text { Average Waiting Time for } \\
\mathrm{QS} \mathrm{HE}_{2} / \mathrm{HE}_{2} / 1\end{array}$ \\
\hline$\rho$ & MAPE & R2Score & MAPE & R2Score \\
\hline 0,1 & 0.063 & 0.983 & 0.0606 & 0.971 \\
\hline 0,5 & 0.0669 & 0.9521 & 0.0094 & 0.9993 \\
\hline 0,9 & 0.0599 & 0.963 & 0.0271 & 0.999 \\
\hline All & 0.0 & 0.9779 & 0.0324 & 0.9993 \\
\hline
\end{tabular}

The results of Evaluation experiments demonstrate that the compared results between simulation and theoretical results are very similar where the Score $\sim=97 \%$ with MAPE $\sim=6 \%$ for $\mathrm{QS}_{2} / \mathrm{H}_{2} /$ 1 and Score $\sim=99 \%$ with MAPE $=3 \%$ for QS $\mathrm{HE}_{2} / \mathrm{HE}_{2} / 1$.

\section{Conclusion}

The goal of this study was to find an approximation for the average waiting time of the queuing system of type $\mathrm{HE}_{2} / \mathrm{HE}_{2} / 1$ using a simulation model Based on Lindley's definition.

The results obtained for the average waiting time are very similar to our theoretical results, from here we can confirm that our results for the approximation of the Average Waiting Time from both the theoretical and simulation results is correct and our proposed simulation model can accurately estimate the other main parameters of the queuing system. The advantage of using simulation is the generating of unlimited data-sets in form of traffic $\operatorname{logs}$, and gives the ability to explore all the states of the queuing system which allows for other studies.

Another advantage is that analyzing the results leads to the detection of possible problems in the system and produce graphical representations which are very handy in the discussion of system refactoring and possible corrections and optimizations.

In the end we say that the obtained results can be used in the optimization of the modern teletraffic systems 


\section{References}

1. L. Kleinrock (1976). Queueing Systems: Theory. Wiley. 448 p.

2. V.N. Tarasov. (2016). Analysis of queues with hyperexponential arrival distributions. Problems of Information Transmission. Vo. 52. No.1, pp.14-23. DOI:10.1134/S0032946016010038

3. V.N. Tarasov. (2018). Analysis and comparison of two queueing systems with hypererlangian input distributions. Radio Electronics, Computer Science, Control. Vol. 47. No.4, pp.61-70. DOI 10.15588/1607-3274-2018-4-6 (in Russian)

4. V.N. Tarasov, E.G. Akhmetshina, O. Kada. (2019). Properties of hyperexponential and hypererlangian distributions. PIC S\&T'2019.

5. Health Services and Delivery Research. No. 3.20. Chapter 5. Bookshelf ID: NBK293948.

6. G/G/1 Queueing Systems, John C.S. Lui.

7. Smmer, Discrete-Event Simulation for R, https://r-simmer.org.
8. distr, Object Oriented Implementation of Distributions. http://distr.r-forge.r-project.org/

9. Roberta Briesemeister and Antônio G.N. Novaes. Comparing an Approximate Queuing Approach withSimulation for the Solution of a Cross-Docking Problem DOI 4987127.

10. Queue Simulation theory,The theoretical study of waiting lines https://www.austincc.edu/akochis/COSC2415/queue-sim.htm.

11. Sanjay K. Bose, Simulation Techniques for Queues and Queueing Networks.

12. Heriot-Watt University, Simulation and Queueing Theory.

13. Jinsung Choi. Simulation of controlled queuing systems and its application to optimal resource management in multiservice cellular networks.

14. J E Beasley, simulation. http://people.brunel.ac.uk/ $\sim$ mastjjb/jeb/or/sim.htm.

\title{
ОПРЕДЕЛЕНИЕ ПРИБЛИЖЕННОГО СРЕДНЕГО ВРЕМЕНИ ОЖИДАНИЯ ДЛЯ СИСТЕМЫ МАССОВОГО ОБСЛУЖИВАНИЯ НЕ2/НЕ2/І С ИСПОЛЬЗОВАНИЕМ МОДЕЛИРОВАНИЯ
}

\author{
Када Отхмане, ПГУТИ, Самара, Россия, otman2333@mail.ru
}

\section{Аннотация}

Представлен симуляционный метод, базирующийся на аппроксимации среднего времени ожидания в системах массового обслуживания, которую Kendall определяет принадлежащей к классам G/G/I, со смешанным вероятностным распределением второй очереди hypererlangian pacпределения для обеих интервальных и сервисных временных функций. Данный метод состоит в создании виртуальной модели загруженности, в первую очередь используя разнообразные методы и алгоритмы для генерации случайных чисел из (hypererlangian) распределения гиперэрланга используя генератор случайных чисел и дискретный симулятор событий, основанный на Системе Очередей (queuing system (QS)) типов HE2/HE2/I, при использовании полученных результатов для анализа поведения системы на различных этапах выполнения. Результаты, полученные для среднего времени ожидания от предложенного метода моделирования, подтверждают теоретические результаты. Таким образом, эта работа позволяет оценить точность теоретических методов, а также собрать большой набор данных, который может быть использован для поиска решения реальных проблем теории телетрафика.

Ключевые слова: дискретный симулятор событий, Система массового обслуживания, распределение гиперэрланга, среднее время ожидания.

\section{Литература}

І. Клейнрок Л. Теория массового обслуживания. 1976. 448 с.

2. Тарасов В.Н. Анализ очередей с гиперэкспоненциальным распределением прихода // Проблемы передачи информации, 2016, т. 5, № 3, № I,

c. 14-23. DOI: 10.1134 / S0032946016010038

3. Тарасов В.Н. Анализ и сравнение двух систем массового обслуживания с гиперэрланговыми входными распределениями // Радиоэлектроника, Информатика, Управление, 20I8, вып. 47, № 4. С. 61-70. DOI 10.15588 / 1607-3274-2018-4-6 (на русском языке)

4. Тарасов В.Н., Ахметшина Е.Г., Када О. Свойства гиперэкспонентного и гиперлерганского распределений // PIC S \& '2019.

5. Исследования в области здравоохранения и доставки, № 3.20, глава 5, ID книжной полки: NBK293948

6. Джон С.С. Луи. G / G / I Queuing Systems.

7. Smmer, моделирование дискретных событий для R, https: //r-simmer.org.

8. distr, объектно-ориентированная реализация распределений. http://distr.r-forge.r-project.org.

9. Роберта Бриземейстер и Антониу Дж. Н. Новаес. Сравнение приближенного подхода к очередям с симуляцией для решения проблемы кроссдокинга. DOI 4987I27

10. Теория имитации очереди, Теоретическое исследование линий ожидания https://www.austincc.edu/akochis/COSC24I5/queue-sim.htm.

II. Санджай К. Бозе. Методы моделирования для очередей и сетей массового обслуживания.

12. Heriot-Watt University, теория моделирования и массового обслуживания.

13. Jinsung Choi Моделирование управляемых систем массового обслуживания и его применение для оптимального управления ресурсами в мультисервисных сотовых сетях.

14. Beasley J.E. Симуляция http://people.brunel.ac.uk/ mastjjb/jeb/or/sim.htm.

Информация об авторе:

Када Отхмане, аспирант, ПГУТИ, Самара, Россия 